\title{
Chiroptical properties of anionic and neutral nickel(II) bis(dithiolene) complexes based on methyl and dimethyl-dddt ligands ${ }^{\dagger}$
}

Alexandre Abhervé, ${ }^{* 1}$ Nabil Mroweh, ${ }^{1}$ Thomas Cauchy, ${ }^{*}, 1$ Flavia Pop, ${ }^{1}$ Nicolas Vanthuyne, ${ }^{2}$ and Narcis Avarvari*,1

${ }^{1}$ Univ Angers, CNRS, MOLTECH-Anjou, SFR MATRIX, F-49000 Angers, France. E-mail: alexandre.abherve@univ-angers.fr; thomas.cauchy@univ-angers.fr; narcis.avarvari@univ$\underline{\text { angers.fr }}$

${ }^{2}$ Aix Marseille Université, CNRS, Centrale Marseille, iSm2, Marseille, France.

${ }^{\dagger}$ Dedicated to Marc Fourmigué on the occasion of his $60^{\text {th }}$ anniversary

\begin{abstract}
Racemic and enantiopure nickel(II) bis(dithiolene) anionic and neutral complexes based on the methyl-5,6-dihydro-1,4-dithiin-2,3-dithiolate (me-dddt) and dimethyl-5,6-dihydro-1,4-dithiin2,3-dithiolate (dm-dddt) ligands have been experimentally and theoretically investigated with a special focus on their chiroptical properties. According to the TD DFT calculations the strong near infrared absorption bands typical for such complexes are only weakly active in CD and, moreover, they have opposite signs for the axial and equatorial conformations, due to the variation of the angle between the transition electric and magnetic dipole moments, thus leading to the mutual cancellation of their contributions and the absence of these bands in the experimental CD spectra. The influence of the number of stereogenic centres and of the oxidation state of the complexes on their chiroptical properties is highlighted. The solid state structure of the complex (TMA)[Ni(rac-me-dddt $\left.)_{2}\right]$ (TMA = tetramethylammonium), determined by single crystal X-ray diffraction analysis, shows a rather unusual cis arrangement of the two dithiolene ligands, with the methyl substituents adopting an axial conformation, which is not the most stable one in the gas phase.
\end{abstract}

Keywords: dithiolene complexes; nickel(II); circular dichroism; DFT calculations; crystalline structures. 


\section{INTRODUCTION}

Chiral transition metal dithiolene complexes represent a particularly attractive class of chiral molecular materials which have been, however, only scarcely investigated so far, mostly for their conducting properties. ${ }^{1,2}$ While the association of chirality with the electron transport properties has been particularly successful in tetrathiafulvalene (TTF) based conductors, ${ }^{3}$ especially through the first evidence of the electrical magneto-chiral anisotropy (eMChA) effect ${ }^{4}$ in bulk crystalline materials, ${ }^{5}$ or the modulation of the crystal packing between racemic and enantiopure forms, ${ }^{6,7}$ the number of conducting chiral metal dithiolene complexes is still limited. They concern, for example, the series of mixed valence $\mathrm{Ni}(\mathrm{dmit})_{2}$ (dmit = 1,3-dithiole2-thione-4,5-dithiolate) anionic complexes with chiral ammonium counter ions, showing semiconducting properties, here the chirality coming exclusively from the cations, ${ }^{8}$ or a series of $\mathrm{Ni}$ (diotte $)_{2}\left(\right.$ diotte $^{2-}$ : dioxolane-dithiocine-dithiolate) anionic complexes with viologen type cations. ${ }^{9}$ Along our own research lines on chiral metal dithiolenes, we have reported the first chiral single component molecular conductor based on neutral enantiopure $\mathrm{Au}(\mathrm{dm}-\mathrm{dddt})_{2}(\mathrm{dm}$ dddt $=$ dimethyl-5,6-dihydro-1,4-dithiin-2,3-dithiolate) complexes ${ }^{10}$ and also the first circularly polarized luminescence (CPL) active metal dithiolene complex containing a [6]helicenedithiolate ligand and a platinum(II) 2,2'-bipy fragment. ${ }^{11}$ More recently, we have investigated the influence of the counter ion and the number of stereogenic centres on the crystal packing and conducting properties of a series of anionic and neutral nickel(II) bis(dithiolene) complexes formulated as $\left[\mathrm{Ni}(\mathrm{dm}-\mathrm{dddt})_{2}\right]^{-1 / 0}$ and $\left[\mathrm{Ni}(\text { me-dddt })_{2}\right]^{-1 / 0}$ (me-dddt $=$ methyl-5,6-dihydro-1,4dithiin-2,3-dithiolate), respectively (Scheme 1). ${ }^{12}$

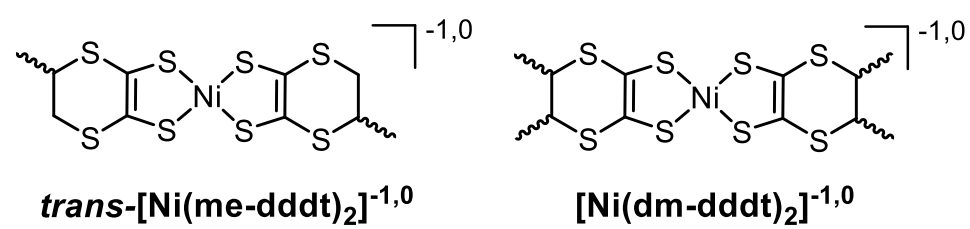

SCHEME 1 Chiral methylated anionic and neutral Ni(II) bis-dithiolene complexes derived from the dddt ligand. Only the trans isomer is shown for $\left[\mathrm{Ni}(\mathrm{me}-\mathrm{dddt})_{2}\right]^{-1 / 0}$

According to our observations, another factor which strongly influences the crystal packing and, consequently, the establishment of intermolecular interactions, is the conformation adopted by the methyl substituents, which can be either axial (ax) or equatorial (eq) (Scheme 2). For example, both all-ax and all-eq conformations were observed in the structures of anionic $(\mathrm{TBA})\left[\mathrm{Ni}(\mathrm{me}-\mathrm{dddt})_{2}\right]$ and $(\mathrm{TBA})\left[\mathrm{Ni}(\mathrm{dm}-\mathrm{dddt})_{2}\right](\mathrm{TBA}=$ tetrabutylammonium $)$, while 
$(\mathrm{TMA})\left[\mathrm{Ni}(\mathrm{dm}-\mathrm{dddt})_{2}\right](\mathrm{TMA}=$ tetramethylammonium $)$ and the neutral complexes $[\mathrm{Ni}(\mathrm{me}-$ dddt $\left.)_{2}\right]$ and $\left[\mathrm{Ni}(\mathrm{dm} \text {-dddt })_{2}\right]$ showed only the all-eq conformation in the solid state. ${ }^{12}$

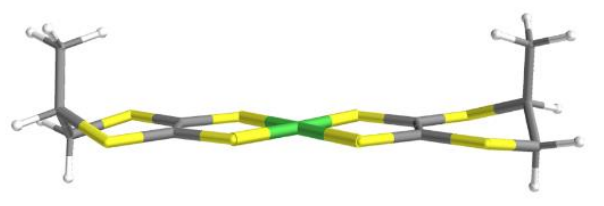

all-ax

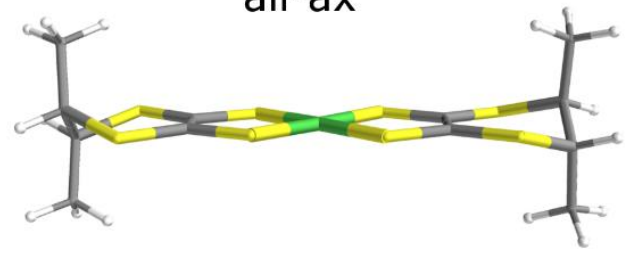

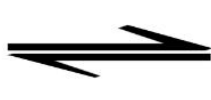
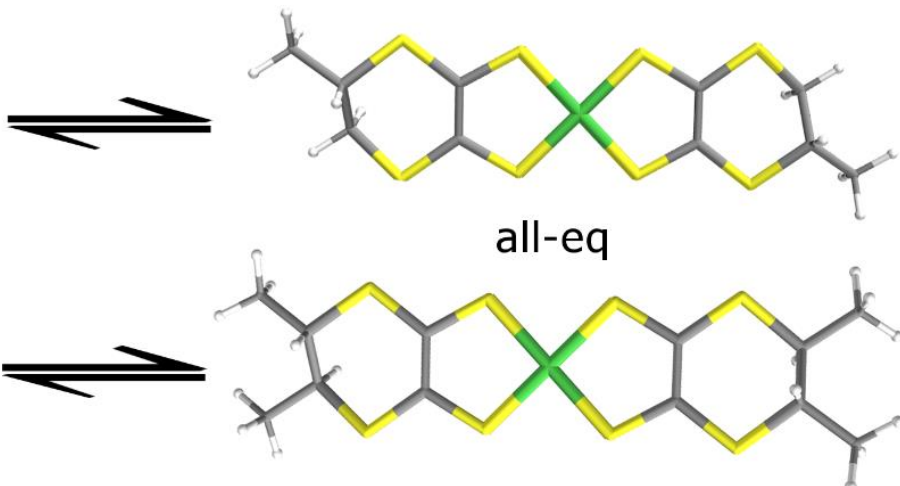

all-eq

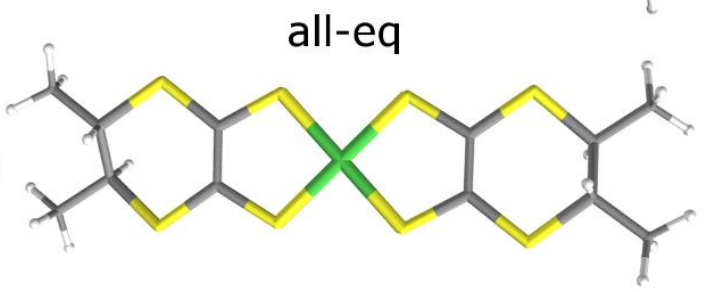

SCHEME 2 Conformational equilibrium in solution for $\left[\mathrm{Ni}(\text { me-dddt })_{2}\right]^{-1 / 0}$ and $\left[\mathrm{Ni}(\mathrm{dm}-\mathrm{dddt})_{2}\right]^{-1 / 0}$. Only the $(S)$ enantiomers are shown, and for $\left[\mathrm{Ni}(\text { me-dddt })_{2}\right]$ only the trans isomer is represented.

However, the two conformations all-ax and all-eq are very likely in fast equilibrium in solution, according to gas phase DFT calculations performed on the thione precursors me-dddt-thione and dm-dddt-thione, which indicate that the eq conformation is slightly more stable by 0.4 $\mathrm{kcal} / \mathrm{mol}$ than the ax one in the former, while the (ax,ax) conformation is more stable by 2.2 $\mathrm{kcal} / \mathrm{mol}$ than the (eq,eq) one in the latter. ${ }^{12}$ Moreover, a fast equilibrium in solution between the (ax,ax) and (eq,eq) conformations was suggested by the calculated interconversion energy barrier of only $\approx 3 \mathrm{kcal} \mathrm{mol}^{-1}$ in the case of the chiral TTF precursor dimethyl-ethylenedithioTTF (DM-EDT-TTF). ${ }^{13}$ On the other hand, the preponderance of one or other conformer was shown to have a massive impact on the chiroptical properties of the thione precursors me-dddt and dm-dddt thiones, ${ }^{12}$ and also on the chiral methylated TTFs DM-EDT-TTF ${ }^{13}$ and TMBEDT-TTF ${ }^{14}$ (tetramethyl-bis(ethylenedithio)-tetrathiafulvalene), since most of the CD bands have inverted signs for the same enantiomer, especially in the lower energy region, when changing from ax to eq. When taking into account that anionic and neutral Ni(II) bis(dithiolene) complexes present very interesting redox switchable optical properties, in particular the existence of strong NIR absorption bands due to HOMO-1 $\rightarrow$ SOMO (SOMO stands for the single occupied molecular orbital with an alpha-spin electron and a beta-spin hole) and HOMO $\rightarrow$ LUMO excitations, ${ }^{15}$ respectively, we decided to investigate the chiroptical properties, both experimentally and theoretically, in the series $\left[\mathrm{Ni}(\mathrm{dm}-\mathrm{dddt})_{2}\right]^{-1 / 0}$ and $\left[\mathrm{Ni}(\text { me-dddt })_{2}\right]^{-1 / 0}$. Note that only a couple of reports deal with chiroptical properties of $\mathrm{Ni}$ (II) bis(dithiolene) complexes, but none of them were supported by theoretical interpretation. Accordingly, Lorcy et al. described chiral $\mathrm{Ni}(\mathrm{II})$ bis(thiazoline-dithiolene) complexes formulated as $[\mathrm{Ni}((R, R)-\mathrm{CHMePh}-$ 
thiazdt $\left.)_{2}\right]^{-2,-1,0}$ and $\left[\mathrm{Ni}\left((S, S)-\mathrm{CHMePh}-\text { thiazdt }_{2}\right]^{-2,-1,0}\right.$ (CHMePh-thiazdt: N-(1-phenylethyl)1,3-thiazoline-2-thione-4,5-dithiolate) for which the circular dichroism spectra have been measured for the three oxidation states. ${ }^{16}$ Interestingly, a very weak CD signal in the NIR region has been observed for the neutral complex. In our previous report on the (TBA)[M(dm-dddt $\left.)_{2}\right]$ and $\left[\mathrm{M}(\mathrm{dm}-\mathrm{dddt})_{2}\right](\mathrm{M}=\mathrm{Ni}, \mathrm{Au})$, the $\mathrm{CD}$ spectra of the anionic complexes in the UV-Vis range have been briefly mentioned. ${ }^{10}$ Finally, Camerel, Fourmigué et al. described a series of neutral $\mathrm{Ni}(\mathrm{II})$ bis(dithiolene) organogelators containing cholesterol fragments which showed solvent dependent NIR CD activity thanks to self-assembly. ${ }^{17}$ In this case, the $\mathrm{CD}$ activity resulted from the supramolecular aggregates and not the molecular species.

We report herein on the redox modulation of the chiroptical properties of $\left[\mathrm{Ni}(\mathrm{dm} \text {-dddt })_{2}\right]^{-1 / 0}$ and $\left[\mathrm{Ni}(\text { me-dddt })_{2}\right]^{-1 / 0}$ supported by TD DFT calculations. The crucial role of the number of stereogenic centres, having a huge impact on the relative stability of the axial and equatorial conformers, will be highlighted.

\section{MATERIALS AND METHODS}

The complexes were obtained as previously reported by us. ${ }^{12}$ MALDI- TOF MS spectra were recorded on Bruker Biflex-IIITM apparatus, equipped with a $337 \mathrm{~nm} \mathrm{~N}_{2}$ laser.

\subsection{CD and UV Measurements}

CD and UV-Vis-NIR absorption spectra were recorded on a Jasco J-1500 CD spectrophotometer equipped with standard PMT detector (for the spectral range 180-800 nm) or InGaAs detector (800-1600 nm). Spectra of (TBA)[Ni(me-dddt) 2 (TBA = tetrabutylammonium) and $(\mathrm{TMA})\left[\mathrm{Ni}(\mathrm{dm}-\mathrm{dddt})_{2}\right](\mathrm{TMA}=$ tetramethylammonium $)$ were measured in $10^{-5} \mathrm{M}$ acetonitrile solutions, while the neutral [Ni(me-dddt $)_{2}$ ] and [Ni(dm-dddt $)_{2}$ ] were recorded in $10^{-5} \mathrm{M}$ dichloromethane and dimethyformamide solutions respectively. All spectra were recorded at $20{ }^{\circ} \mathrm{C}$ in the transmission mode.

\subsection{Single Crystal X-ray Diffraction}

X-ray single-crystal diffraction data were collected on an Agilent SuperNova diffractometer equipped with Atlas $\mathrm{CCD}$ detector and mirror monochromated micro-focus $\mathrm{Cu}-\mathrm{K}_{\alpha}$ radiation $(\lambda$ $=1.54184 \AA$ ). The structure was solved by direct methods, expanded and refined on $\mathrm{F}^{2}$ by full matrix least-squares techniques using SHELX programs (G.M. Sheldrick, 2016). All non-H 
atoms were refined anisotropically and the $\mathrm{H}$ atoms were included in the calculation without refinement. Multiscan empirical absorption was corrected using CrysAlisPro program (CrysAlisPro, Agilent Technologies, V1.171.37.35g, 2014). A summary of the crystallographic data and the structure refinement is given in Table 1. CCDC 2097560 ((TMA)[Ni(rac-medddt)2]) contains the supplementary crystallographic data for this paper. These data can be obtained free of charge from The Cambridge Crystallographic Data Centre via www.ccdc.cam.ac.uk/data_request/cif.

TABLE 1 Crystallographic data, details of data collection and structure refinement parameters

\begin{tabular}{ll}
\hline & (TMA)[Ni(rac -me-dddt) $\left.)_{2}\right]$ \\
\hline Formula & $\mathrm{C}_{14} \mathrm{H}_{24} \mathrm{NS}_{8} \mathrm{Ni}$ \\
$M\left[\mathrm{gmol}^{-1}\right]$ & 521.53 \\
$T[\mathrm{~K}]$ & 150 \\
crystal system & Orthorhombic \\
space group & Pnn2 \\
$a[\AA]$ & $9.6045(3)$ \\
$b[\AA]$ & $10.5325(3)$ \\
$c[\AA]$ & $10.7152(3)$ \\
$V\left[\AA \AA^{3}\right]$ & $1083.94(5)$ \\
$Z$ & 2 \\
$\rho_{\text {calcd }}$ [gcm $\left.^{-3}\right]$ & 1.598 \\
$\mu\left[\mathrm{mm}^{-1}\right]$ & 8.482 \\
$\mathrm{Rint}$ & 0.0252 \\
goodness-of-fit on $\mathrm{F}^{2}$ & 1.088 \\
final R1/ $w 2$ R2 $[\mathrm{I}>2 \sigma(\mathrm{I})]$ & $0.0581 / 0.1552$ \\
$\mathrm{R} 1 / w \mathrm{R} 2($ all data $)$ & $0.0642 / 0.1648$ \\
$\mathrm{CCDC}$ number & 2097560 \\
\hline
\end{tabular}

\subsection{DFT calculations}

All DFT and TD-DFT calculations have been performed with the Gaussian 09 program $^{18}$ using the DFT method with the hybrid PBE0 functional (with $25 \%$ of exact exchange) and the augmented and polarized Ahlrichs triple-zeta basis set (noted TZVP). ${ }^{19}$ Default parameters were used. First, the gas-phase ground state geometries were optimized starting from X-ray diffraction data of (TMA)[Ni(rac-me-dddt $\left.)_{2}\right]$ and previously reported complexes. ${ }^{12} 70$ and 60 low-lying excited-states energies were then determined for the mono-methyl and dimethyl complexes, respectively, for the gas-phase by a linear response TD-DFT method with the same parameters. The calculated spectra have been enlarged by a Gaussian shape [full width at halfmaximum $(\mathrm{FWHM})=3000 \mathrm{~cm}^{-1}$ ] The full reports of the computational results included in the supplementary data were prepared with quchemreport, a homemade python program based on cclib. ${ }^{20,21}$ 


\section{RESULTS AND DISCUSSION}

Racemic and enantiopure forms of the anionic complexes $\left[\mathrm{Ni}(\mathrm{dm}-\mathrm{dddt})_{2}\right]^{-1}$ and $\left[\mathrm{Ni}(\mathrm{me}-\mathrm{dddt})_{2}\right]^{-}$ ${ }^{1}$, which are radical species, with TBA or TMA as counter ions, have been prepared according to our procedures. ${ }^{10,12}$ Then, electrocrystallization of these salts afforded the corresponding closed-shell neutral complexes upon one-electron oxidation of the radical anion precursors. ${ }^{12}$ In our previous reports the solid state structures of the various complexes were described, excepted those of the (TMA)[Ni(me-dddt $\left.)_{2}\right]$ series. We succeeded during the present study to obtain suitable single crystals of (TMA)[Ni( $\mathrm{rac}$-me-dddt $\left.)_{2}\right]$ which could be analysed by single crystal X-ray diffraction analysis.

\subsection{Structure of (TMA)[Ni(rac-me-dddt $\left.)_{2}\right]$}

The compound (TMA)[Ni(rac-me-dddt $\left.)_{2}\right]$ crystallized in the orthorhombic space group Pnn2 with one half Ni complex and one half disordered TMA cation in the asymmetric unit (Figure 1). The metal ion is located on a $C_{2}$ symmetry axis perpendicular to the mean plane of the molecule, which generates the second dithiolene ligand with the same absolute configuration of the carbon atom bearing the methyl substituent (only the $(S)$ configuration is shown in Figure 1) and provides a cis configuration to the complex, with a trans mutual arrangement of the methyl groups. The racemic nature of the complex is confirmed by the twinning of the structure with a Flack parameter of 0.5. The complex presents the all-ax conformation, which is rather unusual in the solid state for metal-bis(dithiolene) complexes based on me-dddt and dm-dddt ligands, where either all-eq or a mixture of all-ax and all-eq conformations were observed. ${ }^{12}$ Table 2 gives a summary of the observed conformations in both TBA and TMA salts of anionic $\left[\mathrm{Ni}(\mathrm{me}-\mathrm{dddt})_{2}\right]$ and $\left[\mathrm{Ni}(\mathrm{dm}-\mathrm{dddt})_{2}\right]$ complexes and in their neutral forms.
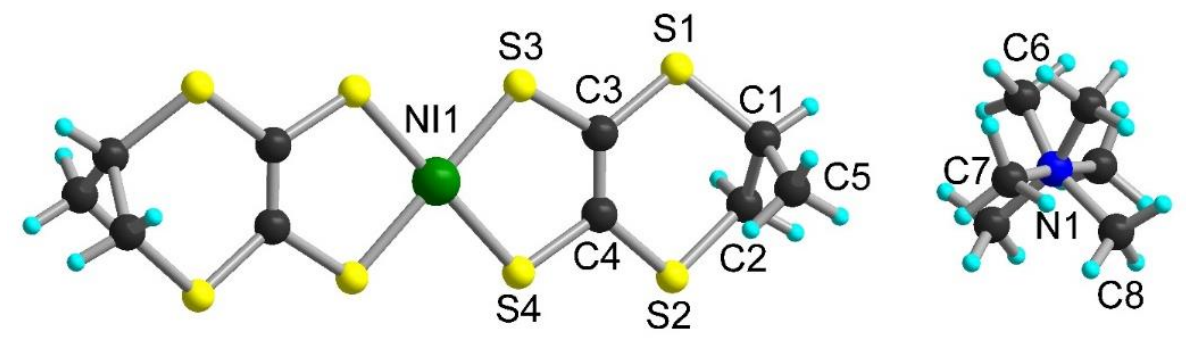

FIGURE 1 Crystal structure of (TMA)[Ni(rac-me-dddt $\left.)_{2}\right]$ with atom label

TABLE 2 Conformation of the methyl substituents in anionic and neutral [Ni(me-dddt $\left.)_{2}\right]$ and $\left[\mathrm{Ni}(\mathrm{dm} \text {-dddt })_{2}\right]$ complexes 


\begin{tabular}{ccc}
\hline (TBA)[Ni(me-dddt $\left.)_{2}\right]$ & $($ TMA $\left.)[\text { Ni(me-dddt })_{2}\right]$ & $\left.[\text { Ni(me-dddt })_{2}\right]$ \\
\hline all-ax / all-eq & all-ax & all-eq \\
\hline (TBA $\left.)[\text { Ni(dm-dddt })_{2}\right]$ & (TMA)[Ni(dm-dddt $\left.)_{2}\right]$ & $\left.[\text { Ni(dm-dddt })_{2}\right]$ \\
\hline all-ax / all-eq & all-eq & all-eq \\
\hline
\end{tabular}

The crystal packing shows a segregation of cations and anions leading to one-dimensional (1D) stacking of the anionic complexes along the $a$ direction (Figures 2 and S1). Similarly to the crystal structures of the non-substituted achiral (TMA)[Ni(dddt) 2$]$ previously reported by Bereman et $a l .{ }^{22}$ and the series of (TMA) $\left[\mathrm{Ni}(\mathrm{dm}-\mathrm{dddt})_{2}\right],{ }^{12}$ the use of the smallest TMA cation leads to a partial overlap of the anionic complexes. However the unusual all-ax conformation of the methyl substituents in (TMA)[Ni( $r a c$-me-dddt $\left.)_{2}\right]$ prevents strong intermolecular $\mathrm{S} \cdots \mathrm{S}$ interactions but rather favours $\mathrm{C}-\mathrm{H} \cdots \mathrm{S}$ contacts of about 3.90(3) $\AA$ between dithiolene and methyl substituents along the $a$ direction. In addition, the existence of this conformation results in a distance of $4.93 \AA$ between two consecutives planes of complexes, larger than the distances observed in (TMA)[Ni(dddt) 2 (about $4.59 \AA$ ) and between the all-eq conformers in (TMA)[Ni(dm-dddt) 2 ] where this distance falls to $4.02 \AA$ (Figure S2). On the other side, one can observe numerous S $\cdots \mathrm{S}$ interactions (of 3.799(6) and 3.814(7) $\AA$ ) between complexes belonging to different 1D chains, as previously observed in (TMA)[Ni(dddt) 2 (Figure S3).

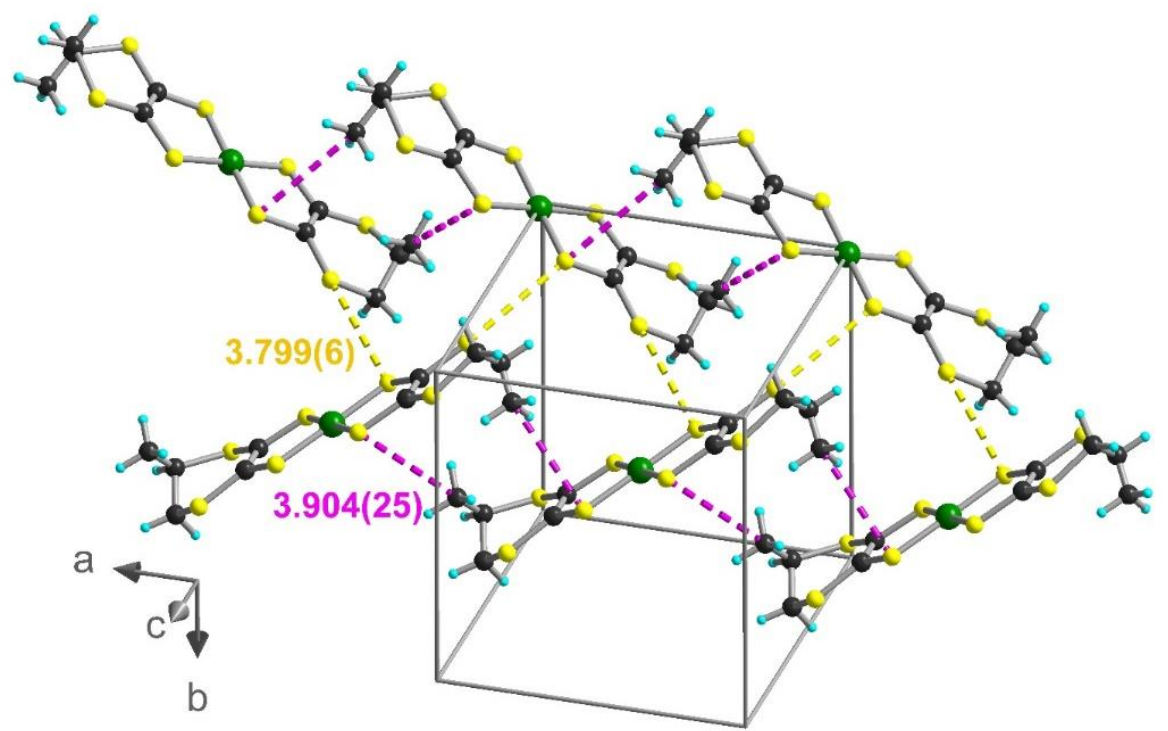

FIGURE 2 Crystal packing of the anionic complexes in (TMA)[Ni(rac-me-dddt) 2 ]. Colour code: $\mathrm{C}$ (black), $\mathrm{H}$ (cyan), S (yellow), Ni (green). Intermolecular $\mathrm{C}-\mathrm{H} \cdots \mathrm{S}$ interactions are represented in pink dashed lines, $\mathrm{S} \cdots \mathrm{S}$ interactions in yellow dashed lines. TMA cations are omitted for clarity

\subsection{UV-Vis and CD spectroscopy}

As outlined in the Introduction, the main purpose of this study relies on the chiroptical investigations of these series of chiral nickel bis(dithiolene) complexes in order to disclose the 
role and influence of the charge and of the number of stereogenic centres. Previously, optical and chiroptical characterization has been performed on the enantiopure (TBA)[Ni(dm-dddt $\left.)_{2}\right]$ complexes. ${ }^{10}$ Here, we checked the enantiopurity and absolute configuration of the anionic complexes (TBA)[Ni(me-dddt $\left.)_{2}\right]$ and (TMA)[Ni(dm-dddt $\left.)_{2}\right]$ by circular dichroism (CD) measurements in solution, mirror-image spectra being obtained for the two enantiomers in both cases (Figure 3).

a)
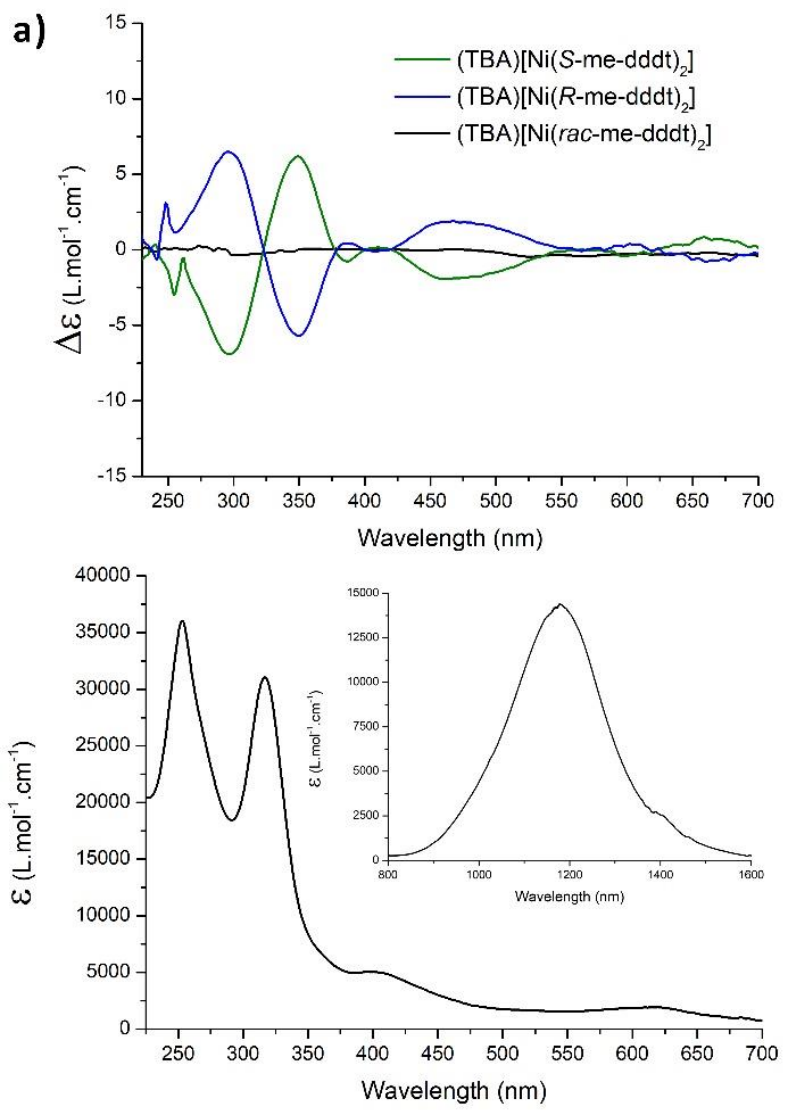

b)
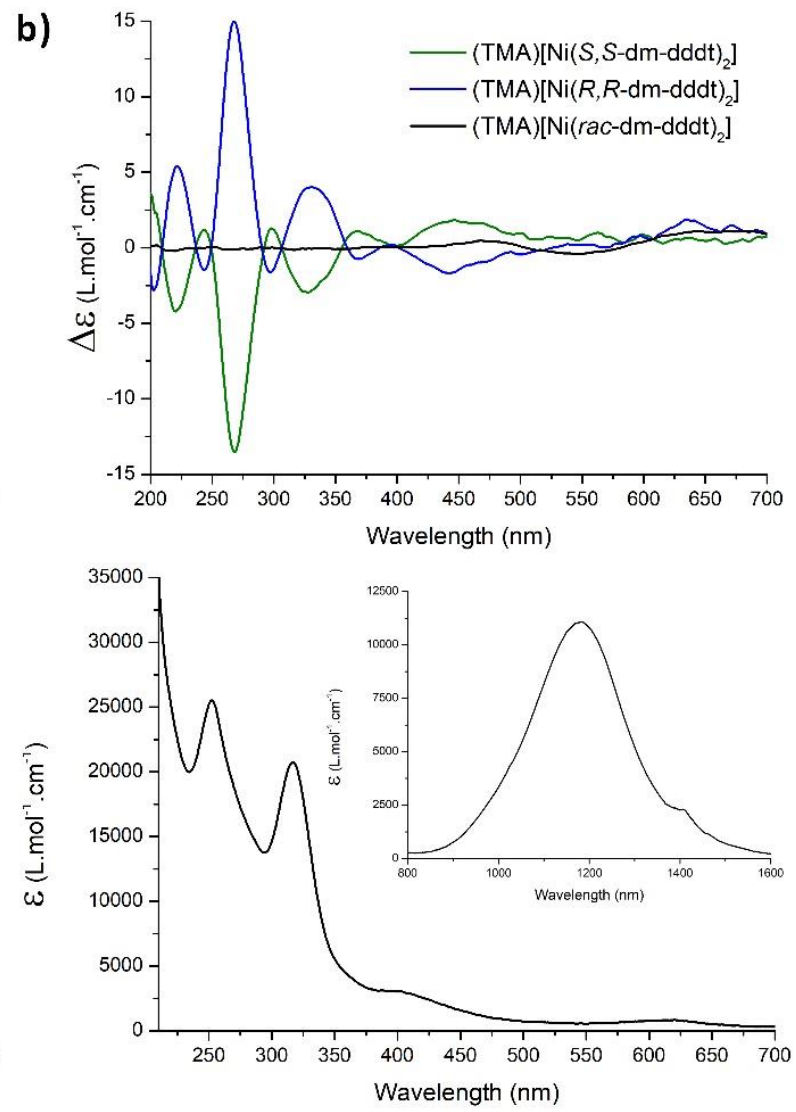

FIGURE $3 \mathrm{CD}$ (top) and UV-Vis spectra (bottom) of (TBA)[Ni(me-dddt) ${ }_{2}$ (a) and (TMA)[Ni(dm-dddt) 2 (b). The insets in the bottom panels show absorption spectra in the NIR region.

As expected, the CD spectra of (TBA) $\left[\mathrm{Ni}(\mathrm{dm}-\mathrm{dddt})_{2}\right]^{10}$ and (TMA)[Ni(dm-dddt $\left.)_{2}\right]$ (Figure $3 \mathrm{~b}$ ) are similar and thus independent of the cation. Although the neutral complexes have been obtained as single crystals by electrocrystallization, their optical and chiroptical properties in solution have been investigated as well upon dissolution of the crystalline materials. The main feature of the UV-Vis-NIR spectra of such neutral complexes is the presence of a very intense band in the NIR region due to the HOMO-LUMO transition, ${ }^{15}$ both orbitals being ligand centred. This band is hypsochromically shifted when compared to the corresponding monoanionic complexes, since the HOMO-1-SOMO transition in the latter occurs at lower energies. Here indeed, we observe, for example, a very intense band at $\lambda_{\max }=1028 \mathrm{~nm}(\varepsilon=$ 
$34600 \mathrm{~L} \mathrm{~mol}^{-1} \mathrm{~cm}^{-1}$ ) for [Ni( $\mathrm{rac}$-me-dddt) 2 (Figure S4), to be compared with the NIR band at $\lambda_{\max }=1176 \mathrm{~nm}\left(\varepsilon=14300 \mathrm{~L} \mathrm{~mol}^{-1} \mathrm{~cm}^{-1}\right)$ shown by (TBA)[Ni( $\mathrm{rac}$-me-dddt) $\left.{ }_{2}\right]$ (inset Figure $3 \mathrm{a}$ bottom). Moreover, mirror-image CD spectra have been observed for both anionic (TBA) $[\mathrm{Ni}(S-$ me-dddt $\left.)_{2}\right]$ and $(\mathrm{TBA})\left[\mathrm{Ni}(R \text {-me-dddt })_{2}\right]$ (Figure $\left.3 \mathrm{a}\right)$ and neutral $\left[\mathrm{Ni}(S \text {-me-dddt })_{2}\right]$ and $[\mathrm{Ni}(R$ me-dddt $)_{2}$ (Figure S5). Furthermore, $(\mathrm{TMA})\left[\mathrm{Ni}(\mathrm{rac}-\mathrm{dm}-\mathrm{dddt})_{2}\right]$ presents a NIR band at $\lambda_{\max }=$ $1185 \mathrm{~nm}\left(\varepsilon=11100 \mathrm{~L} \mathrm{~mol}^{-1} \mathrm{~cm}^{-1}\right)$ (inset Figure $3 \mathrm{~b}$ bottom) which is more intense and blueshifted at $\lambda_{\max }=1051 \mathrm{~nm}\left(\varepsilon=40800 \mathrm{~L} \mathrm{~mol}^{-1} \mathrm{~cm}^{-1}\right)$ in the neutral complex [Ni(rac-dm-dddt $\left.)_{2}\right]$ (Figure S6). Mirror-image CD spectra were also observed for the neutral complexes $[\mathrm{Ni}(S, S$ $\mathrm{dm}$-dddt $\left.)_{2}\right]$ and $\left[\mathrm{Ni}(R, R \text {-dm-dddt })_{2}\right]$ (Figure $\left.\mathrm{S} 7\right)$. As a general feature, it can be clearly observed that the CD bands are more intense for the dm-dddt based complexes than those for me-dddt ones for both anionic and neutral series. Moreover, massive differences concerning the signs of some of the strongest bands can be noticed between dm-dddt and me-dddt complexes with the same configurations, i.e. $(S, S)$ and $(S)$, respectively. For example, the strongest CD band in the visible region at $\approx 450 \mathrm{~nm}$ is positive for the former and negative for the latter. These differences, also observed for the ligands precursors dm-dddt-thione and me-dddt-thione, ${ }^{12}$ most probably find their origin in the different stability of the axial and equatorial forms of the two ligands. Rather surprisingly at a first sight, no CD bands could be detected in the NIR region for any of the investigated complexes. In order to shed light on the chiroptical properties of these chiral nickel bis(dithiolene) complexes, a theoretical study has been undertaken.

\subsection{DFT and TD-DFT calculations}

DFT and TD-DFT calculations have been carried out for the compounds $\left[\mathrm{Ni}(\text { me-dddt })_{2}\right]^{-1}$ and $\left[\mathrm{Ni}(\mathrm{dm}-\mathrm{dddt})_{2}\right]^{-1}$, open-shell, and $\left[\mathrm{Ni}(\text { me-dddt })_{2}\right]^{0}$ and $\left[\mathrm{Ni}(\mathrm{dm}-\mathrm{dddt})_{2}\right]^{0}$, close-shell. They were investigated for both enantiomers as axial and equatorial conformations (see computational details and Figures S8-S98). Moreover, for the mono-methyl derivative of the radical anion species, both trans and cis isomers have been considered. The gas phase calculated energies confirm a weakly favoured equilibrium for the equatorial forms of the mono-methyl compounds, while for di-methylated complexes the axial conformations are the most stable and clearly predominant (Table 3 ), in line with the previous results on the dithiolene precursors. ${ }^{12}$ Note that, in the case of the me-dddt complexes, the higher stability, albeit weak, of the equatorial conformer compared to the axial one was found for both trans and cis isomers, which very likely interconvert in solution and thus contribute to the experimentally observed CD. 
TABLE 3 Gibbs Free Energies differences in $\mathrm{kcal} / \mathrm{mol}$ between the axial and equatorial conformations and their expected ratios in gas phase and room temperature

\begin{tabular}{ccc}
\hline Compound & $\Delta \mathrm{G}($ ax-eq $)$ & $\begin{array}{c}\text { RT ax : eq } \\
\text { gas phase ratio }\end{array}$ \\
\hline trans $\left[\mathrm{Ni}(R \text {-me-dddt })_{2}\right]^{-}$ & 0.5 & $1: 2.5$ \\
cis $\left[\mathrm{Ni}(R \text {-me-dddt })_{2}\right]^{-}$ & 1.0 & $1: 5$ \\
{$\left[\mathrm{Ni}(R \text {-me-dddt })_{2}\right]$} & 0.9 & $1: 5$ \\
{$\left[\mathrm{Ni}(R, R \text {-dm-dddt })_{2}\right]^{-}$} & -1.9 & $26: 1$ \\
{$\left[\mathrm{Ni}(R, R \text {-dm-dddt })_{2}\right]$} & -0.7 & $3: 1$ \\
\hline
\end{tabular}

If one considers, for example the case of $\left[\mathrm{Ni}(R \text {-me-dddt })_{2}\right]^{-1}$, for which the equatorial conformation is slightly favoured by $0.5 \mathrm{kcal} \mathrm{mol}^{-1}$, the theoretical CD spectrum should, in principle, result from a Boltzmann analysis of the CD spectra of the axial and equatorial forms, which present almost the same bands but with opposite signs (see the SI). Accordingly, such a simulation, undertaken here for the trans isomer, clearly shows that bands which are already weakly intense in the pure axial and equatorial conformations, as the ones in the NIR region, tend to cancel (Figure 4). It is thus not surprising that for our chiral complexes, showing a dynamic behaviour in solution, the CD NIR bands cannot be observed.

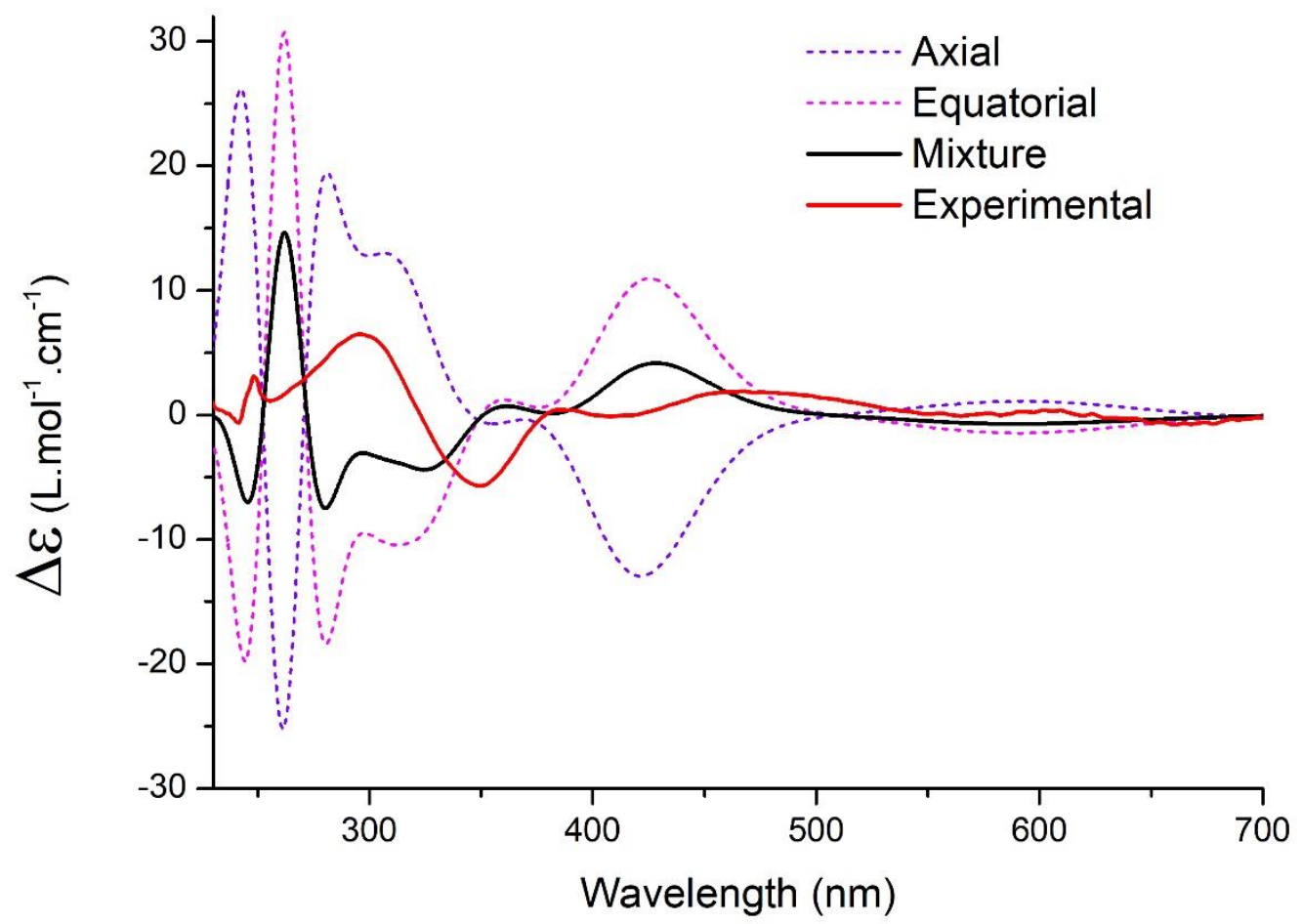

FIGURE 4 Simulated CD spectra (dashed lines) for axial and equatorial conformations in $\left[\mathrm{Ni}(R \text {-me-dddt })_{2}\right]^{-1}$. The black curve corresponds to the resulting expected spectrum according to the Boltzmann analysis of the calculated gas phase ratio, and the red curve corresponds to the experimental spectrum in solution. 
For example, when considering the two most active $\mathrm{CD}$ transitions of $\left[\mathrm{Ni}(R \text {-me-dddt })_{2}\right]^{-1}$ in the NIR region, that is S3 and S6 (see the SI), it can be clearly seen that they occur practically at the same energy, i.e. $\approx 1150$ and $\approx 980 \mathrm{~nm}$, and have roughly the same intensity but with opposite signs (Figure 5).

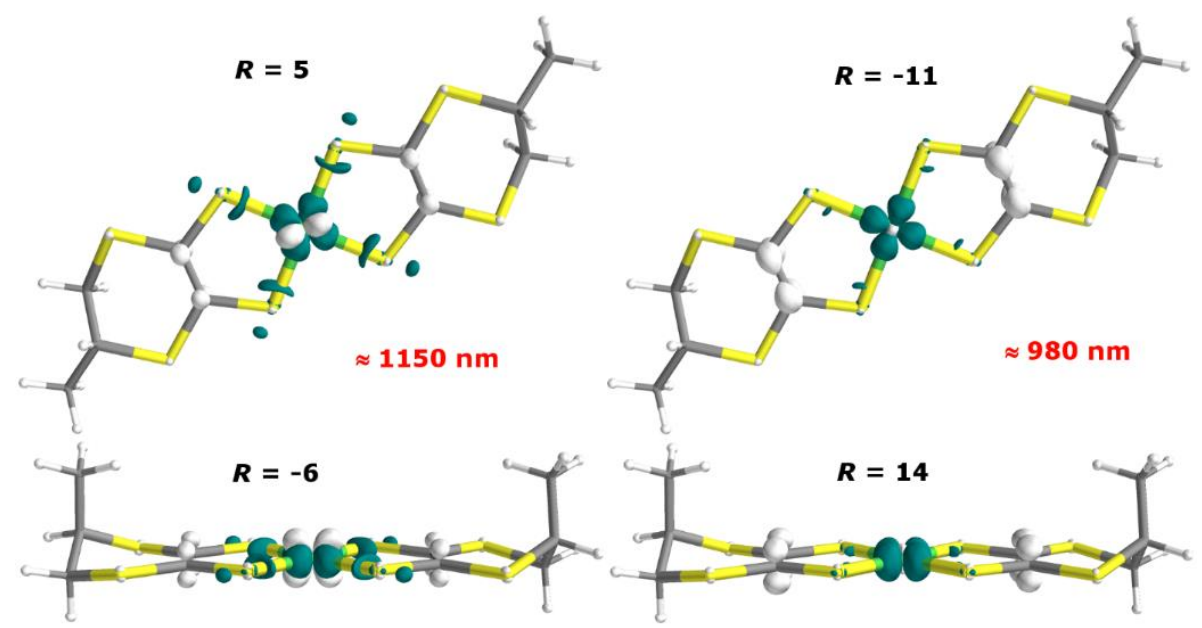

FIGURE 5 Representation of the $\mathrm{S} 3(\mathrm{eq}) / \mathrm{S} 4(\mathrm{ax})$ and $\mathrm{S} 6$ transitions for the monoanionic complex $[\mathrm{Ni}(R$-me$\left.\mathrm{dddt}_{2}\right]^{-1}$ in both equatorial (top) and axial conformations (bottom). The excited electron and the hole regions are indicated by respectively blue and white surfaces

While the S3/S4 excitation is almost absent in the absorption spectrum since it is electric dipole symmetry forbidden, the S6 excitation corresponds to the intense HOMO-1 $\rightarrow$ SOMO transition in the NIR region, usually observed for all radical anions of nickel(II) bis(dithiolene) complexes. However, since it involves a $\pi-\pi^{*}$ excitation, the magnetic dipole transition is much weaker. Moreover, the analysis of the electric and magnetic dipole moments of this transition reveals that the angle between them changes from $104.5^{\circ}$ in the equatorial conformer to $75.1^{\circ}$ in the axial one, leading to similar absolute values of the rotational strength but opposite signs (Table 4). As a consequence, for such symmetric bis(dithiolene) complexes showing such conformational equilibrium the NIR band can never be very intense.

TABLE 4 Electric and Magnetic dipole angle in velocity gauge for the S6 transition of $\left[\mathrm{Ni}(R \text {-me-dddt })_{2}\right]^{-}$

\begin{tabular}{ccc}
\hline conformer & $\mu^{*}-\mathrm{m}^{*}$ angle $\left(^{\circ}\right)$ & $\mathrm{R}$ (velocity, length) \\
\hline equatorial & 104.5 & $-9.8,-10.9$ \\
axial & 75.1 & $12.7,13.9$ \\
\hline
\end{tabular}


For the corresponding neutral complex $\left[\mathrm{Ni}(R \text {-me-dddt })_{2}\right]$, the all-ax conformer should have a $\mathrm{CD}$ band at $828 \mathrm{~nm}$ corresponding to a HOMO $\rightarrow$ LUMO excitation (S1) with a positive rotational strength $R=15.3$, whereas the same band, appearing at $827 \mathrm{~nm}$, is negative $(\mathrm{R}=$ 15.3) for the all-eq conformer (see the SI).

In reality the situation is even more complex because of the structural flexibility of the ligands and complexes and of the small energy differences between all the possible stable forms, since in solution the mixed conformation, with one ligand as axial conformer and the second ligand as equatorial conformer, can occur as well. Moreover, in the case of the me-dddt complexes the cis isomers are also present, being in equilibrium with the trans forms, as shown by scrambling experiments $^{12}$ and by the crystallization of the cis isomer for (TMA)[Ni(rac-me-dddt) 2 (vide supra).
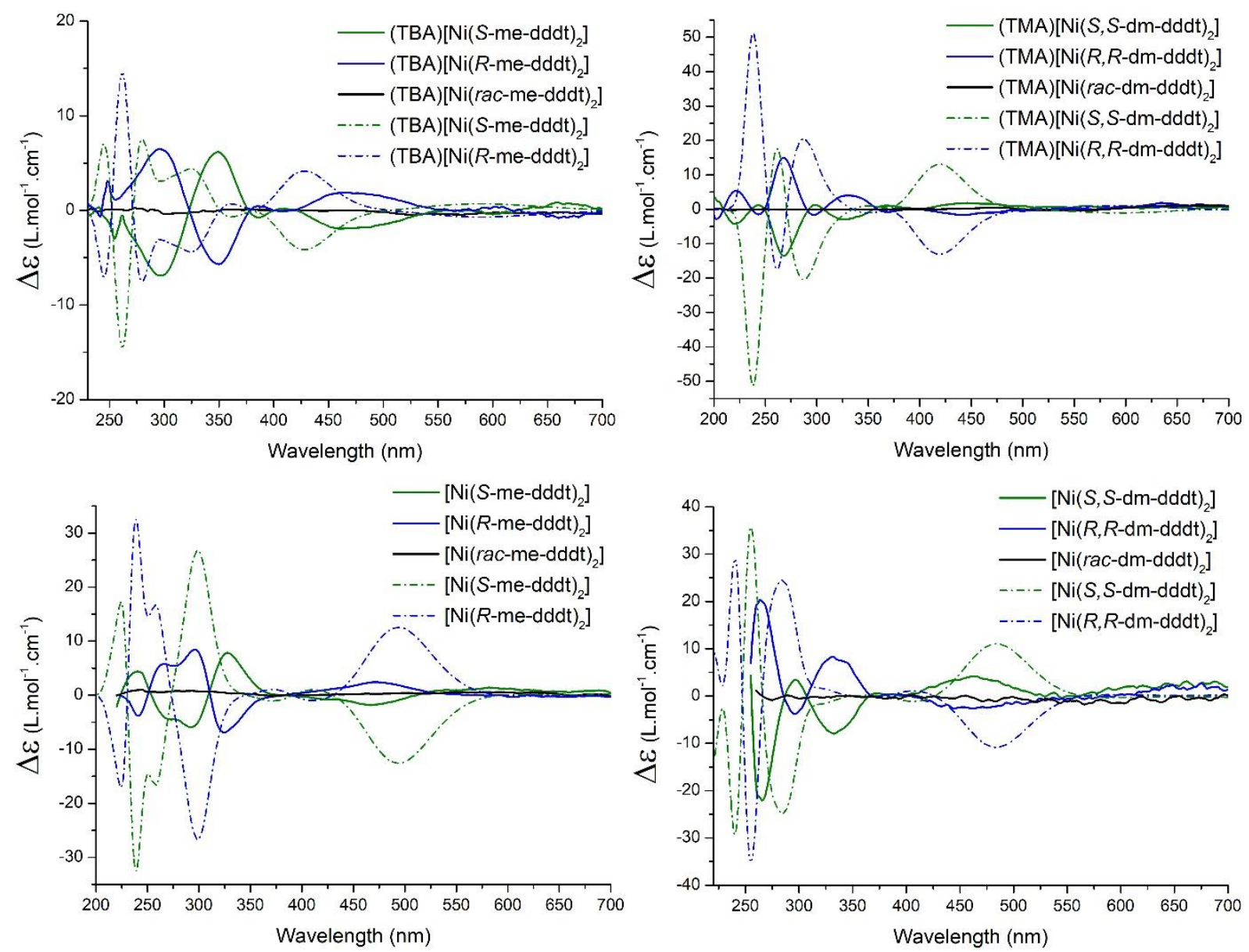

FIGURE 6 Experimental (solid lines) and simulated CD spectra (dashed-dotted lines) for the anionic (top) and neutral (bottom) complexes

However, the agreement between the experimental and simulated CD spectra for the four series of complexes is very good (Figure 6), especially for the dm-dddt based complexes (right panels 
in Figure 6), probably because of the absence of cis/trans isomers, further complicating the analysis in the case of me-dddt complexes. Generally a slight blue-shift is observed for the calculated transitions compared to the experimental ones, as also noticed for the dithiolene precursors, ${ }^{12}$ excepting for the low energy band calculated at around $490 \mathrm{~nm}$ for the neutral complexes (bottom panels in Figure 6, see also the SI), yet the overall shapes of the respective experimental and simulated spectra accurately match. The analysis of these low energy bands reveals that they involve transitions between molecular orbitals containing non-negligible contributions of $\mathrm{Ni}$ (II) d orbitals (see the SI), thus highlighting the importance of the metal centre for the chiroptical properties of such chiral square planar metal bis(dithiolene) complexes.

\section{CONCLUSION}

In this study we have investigated experimentally and theoretically the optical and chiroptical properties of anionic and neutral chiral nickel(II) bis(dithiolene) complexes based on the methyl-5,6-dihydro-1,4-dithiin-2,3-dithiolate (me-dddt) and dimethyl-5,6-dihydro-1,4-dithiin2,3-dithiolate (dm-dddt) ligands, highlighting the crucial role of the number of stereogenic centres in the chiroptical properties through the preferential occurrence of axial or equatorial conformations of the methyl substituents. The first sight surprising experimental absence of CD active NIR bands for both anionic and neutral series in spite of their strong intensity in the UVvisible-NIR absorption spectra, finds its origin in the mutual cancellation of the opposite in sign contributions of the axial and equatorial conformers. This represents the first combined experimental/theoretical chiroptical study of chiral metal bis(dithiolene) complexes, allowing to finely disclose the importance of the conformational issues and of the oxidation state in the chiroptical properties of such materials. Future work will be devoted to the preparation of more rigid chiral structures in order to avoid the cancellation of the $\mathrm{CD}$ bands through conformational equilibrium.

\section{ACKNOWLEDGMENTS}

This work was supported in France by the National Agency for Research (ANR, Project 15CE29-0006-01 ChiraMolCo), the CNRS and the University of Angers.

\section{DATA AVAILABILITY STATEMENT}


The data that support the findings of this study are available from the corresponding authors upon reasonable request.

\section{REFERENCES}

1. Pop F, Zigon N, Avarvari N. Main-Group-Based Electro- and Photoactive Chiral Materials. Chem Rev. 2019;119:8435-8478.

2. Pop F, Avarvari N. Chiral metal-dithiolene complexes. Coord Chem Rev. 2017;346:20-31.

3. Avarvari N, Wallis JD. Strategies towards chiral molecular conductors. J Mater Chem. 2009;19:4061-4076.

4. Krstić V, Roth S, Burghard M, Kern K, Rikken GLJA. Magneto-chiral anisotropy in charge transport through single-walled carbon nanotubes. J Chem Phys. 2002;117:11315-11319.

5. Pop F, Auban-Senzier P, Canadell E, Rikken GLJA, Avarvari N. Electrical magneto-chiral anisotropy in a bulk chiral molecular conductor. Nat Commun. 2014;5:3757.

6. Pop F, Auban-Senzier P, Frąckowiak A, Ptaszyński K, Olejniczak I, Wallis JD, Canadell E, Avarvari N. Chirality Driven Metallic versus Semiconducting Behavior in a Complete Series of Radical Cation Salts Based on Dimethyl-Ethylenedithio-Tetrathiafulvalene (DM-EDT-TTF). J Am Chem Soc. 2013;135:1717617186.

7. Pop F, Auban-Senzier P, Canadell E, Avarvari N. Anion size control of the packing in the metallic versus semiconducting chiral radical cation salts (DM-EDT-TTF $)_{2} \mathrm{XF}_{6}(\mathrm{X}=\mathrm{P}, \mathrm{As}$, Sb). Chem Commun. 2016;52:12438-12441.

8. Lieffrig J, Jeannin O, Auban-Senzier P, Fourmigué M. Chiral Conducting Salts of Nickel Dithiolene Complexes. Inorg Chem. 2012;51:7144-7152.

9. Kisch H, Eisen B, Dinnebier R, Shankland K, David WIF, Knoch F. Chiral Metal-Dithiolene/Viologen Ion Pairs: Synthesis and Electrical Conductivity. Chem Eur J. 2001;7:738-748.

10. Branzea DG, Pop F, Auban-Senzier P, Clérac R, Alemany P, Canadell E, Avarvari N. Localization versus Delocalization in Chiral Single Component Conductors of Gold Bis(dithiolene) Complexes. J Am Chem Soc. 2016;138:6838-6851.

11. Biet T, Cauchy T, Sun Q, Ding J, Hauser A, Oulevey P, Bürgi T, Jacquemin D, Vanthuyne N, Crassous J, Avarvari, N. Triplet State CPL Active Helicene-Dithiolene Platinum Bipyridine Complexes. Chem Commun. 2017;53:9210-9213.

12. Abhervé A, Mroweh N, Cauchy T, Pop F, Cui H, Kato R, Vanthuyne N, Alemany P, Canadell E, Avarvari, N. Conducting chiral nickel(II) bis(dithiolene) complexes: structural and electron transport modulation with the charge and the number of stereogenic centres. J Mater Chem C. 2021;9:4119-4140.

13. Cauchy T, Pop F, Cuny J, Avarvari N. Conformational Study and Chiroptical Properties of Chiral DimethylEthylenedithio-Tetrathiafulvalene (DM-EDT-TTF). Chimia. 2018;72:389-393.

14. Pop F, Laroussi S, Cauchy T, Gómez-García CJ, Wallis JD, Avarvari N. Tetramethyl Bis(ethylenedithio)Tetrathiafulvalene (TM-BEDT-TTF) Revisited: Crystal Structures, Chiroptical Properties, Theoretical Calculations and a Complete Series of Conducting Radical Cation Salts. Chirality. 2013;25:466-474. 
15. Lim BS, Fomitchev DV, Holm RH. Nickel Dithiolenes Revisited: Structures and Electron Distribution from Density Functional Theory for the Three-Member Electron-Transfer Series $\left[\mathrm{Ni}\left(\mathrm{S}_{2} \mathrm{C}_{2} \mathrm{Me}_{2}\right)_{2}\right]^{0,1-2-}$. Inorg Chem. 2001;40:4257-4262.

16. Le Gal Y, Vacher A, Dorcet V, Fourmigué M, Crassous J, Lorcy D. The near infra red (NIR) chiroptical properties of nickel dithiolene complexes. New J Chem. 2015;39:122-129.

17. Debnath S, Bergamini J-F, Artzner F, Mériadec C, Camerel F, Fourmigué M. Near-infrared chiro-optical effects in metallogels. Chem Commun. 2012;48:2283-2285.

18. Frisch MJ et al., Gaussian 09 Revision D.01.

19. Schäfer A, Huber C, Ahlrichs R. Fully optimized contracted Gaussian basis sets of triple zeta valence quality for atoms Li to Kr. J Chem Phys 1994;100:5829-5835.

20. Cauchy T, Da Mota B. Quchemreport. A python program for control quality and automatic generation of quantum chemistry results. University of Angers, 2020.

21. O’boyle NM, Tenderholt AL, Langner KM. cclib: A library for package-independent computational chemistry algorithms. J Comput Chem 2008;29:839-845.

22. Welch JH, Bereman RD, Singh P, Haase D, Hatfield WE, Kirk ML. Effect of Cation on the Structures, Magnetic Properties and Specific Heats of Salts of Bis(5,6-dihydro-1,4-dithiin-2,3dithiolato)nickelate(III): the Crystal Structure of $\left[\left(\mathrm{CH}_{3}\right)_{4} \mathrm{~N}\right]\left[\mathrm{Ni}(\mathrm{DDDT})_{2}\right]$. Inorg Chim Acta 1989;162:89-96. 